\title{
WOMEN'S AUTONOMY AND UTILIZATION OF MATERNAL HEALTH CARE SERVICES IN RURAL NEPAL
}

\begin{abstract}
Kamala Lamichhane*
Nepal has one of the highest maternal mortality ratios in South Asia although the situation is improving in recent days. One of the reasons for such a high mortality may be attributed to the under-utilization of modern maternal health services during pregnancy. This study has analyzed the relationship between women's autonomy and the utilization of maternal health care services in order to explore some possible reasons for the under-utilization of the services. Data from the Nepal demographic and Health Survey, 2011 is used for the study. The analysis is based on 2374 married women aged 15-49 years who had given a live birth during three years preceding the survey. Women's household decision making power, control over use of earning and decision on using contraception have been used to explore the indicators of women's autonomy. Logistic regression is used to assess the net effect of several independent variables on two dependent variables (adequate antenatal care and institutional delivery) of maternal health care. Logistic regression analyses reveal that the utilization of both maternal health care services are related to women's autonomy as indicated by decision making power on own health care, large household purchases and control over earnings because financial sufficiency is must at that period. Women's' decision-making power appears to be the most powerful predictors for increasing maternal health service utilization. The study results suggest that policy actions that increase women's autonomy at home could be effective in helping assure good maternal health.
\end{abstract}

Keywords: Autonomy, rural women and maternal health.

\section{INTRODUCTION}

Maternal health is an important part of the health care system aimed at reducing morbidity and mortality related to pregnancy. The health care that a woman receives during pregnancy, at the time of delivery, and soon after delivery is important for the survival and well-being of both the mother and the child (MoHP, New ERA, \& Macro International Inc., 2007). Maternal health seeking behaviour is different according to different society, culture, caste and class of people. However, in developing countries pregnancy and child birth are the leading cause of death among women of reproductive age. Poverty, cultural factors that restrict women's autonomy, promote early marriage or support harmful traditional practices, nutritional deficiencies, reproductive factors such as young age at first birth, distance to health services, and inadequate health services are the determinants of poor maternal outcomes.

'Status', 'Empowerment', and 'Autonomy', are synonymously used in the literature on gender to capture the degree of access, control and independence in decision-making by women. Status is position, condition or standing. It is subjective. Attaining status neither ensures decision making ability nor autonomy in women. Autonomy' is a construct which explains the status in which a person has control over the activities relating own lives (wellbeing). It emphasizes the capacity to make decisions, use of resources and ability to meet requirements of self and the immediate others on one's own. Empowerment is relatively dynamic and considers the process (Batliwala, 1994)

* Ms. Lamichhane is Lecturer at Central Department of Population Studies (CDPS), Tribhuvan University, Kirtipur, Kathmandu, Nepal. 
whereas autonomy reflects more static position irrespective of process (Jejeebhoy, 2000a). Women's autonomy as individuals is conditioned largely by the extent of gender stratification prevailing in their society (Jejeebhoy, 2000b).

In communities, especially in patriarchal and patrilocal, the autonomy is inherited to male members and females are excluded (Raddicliff-Brown, 1952). In such cultures, gender relations are inegalitarian and women have little say in their own lives, and thus, limited autonomy. This tendency operates throughout the life course in a downward spiral unless some external forces operate to break the cycle.

In Nepal low social status of women has been identified as a hindrance to progress towards national health and population policy targets. Although it seems reasonable to assume that women's autonomy (status) within the household leads to higher use of maternal health care services, this factor has not been explore for Nepal. We know little about how intra household relation constrain or facilitate access to health care, or about the dimension of women's position that are most critical for achieving increased use.

The fundamental theory of maternity care is to ensure the life of the mother and her newborn child by providing comfort and welfare for an individual, family and community society along with the policy makers of the country as fully as possible. For this purpose, MOHP has formulated robust sets of policies such as National Health Policy (MoHP, 1991) and Safe Motherhood Policy (MoHP, 1994) National Maternity Care Guidelines, Nepal (MoHP, 1996) to ensure maternity care in each corner of the country.

Maternal mortality in Nepal is estimated to be around 281 deaths per 100,000 live births (MoHP, New ERA, \& Macro International Inc., 2007). One major factor is low use of maternal health care services. In seeking to explain these low level of health care use, most research has focused on the provision and geographic accessibly of services. However no studies have looked at how sociocultural factures such as inequality, gender roles and women's autonomy within the household have influenced use of services. This relationship is particularly critical for countries, where gender disparity still is large. The role of women's autonomy in maternal health is important to save their own lives and their children's lives. Thus, this study intends to fill this gap and to contribute to the understanding of the numerous interconnections between women's autonomy and utilization of maternal health care.

Although, there are perceived influences of women's improved status in reproductive behaviours, empirical evidences to approve this influences are considerably lacking. A research gap is observed whether there is an observable links between women's autonomy and utilization of maternal health care in rural Nepal. There are also arguments and counterarguments in the improvement of women's status which has been examined in this study using data from 2011 NDHS. Additionally, women's autonomy is influenced by context specific variables. This study has consider indirect independent contextual factors (such asage, age at marriage, birth planning status, number of living children, education of women, education of husband, occupation of women, household economic status, and exposure to media) and its influence on maternal health services.

\section{DATA AND METHOD}

\section{Source of data}

The data for this study has been based on 2011 Nepal Demographic and Health Survey. This survey included questions related to fertility, family planning, infant and child mortality, maternal 
and child health, breastfeeding and nutrition, HIV/AIDS and STIs, along with information on women's status and decision-making power in the family that allows us to explore the relationship between women's autonomy and maternal health care services utilization in Nepal.

The sample used for this study consists of rural women who had at least one birth in the three year before the survey. And only utilization behaviour associated with the most recent pregnancy was considered. The sample was selected with the rationale that mothers would most accurately be able to recall the utilization behaviour of their pregnancy that occurred within the three year. The analysis was also limited to rural women listed as usual place of residents of the community in which they were surveyed to avoid ascribing local contextual factors to women who did not normally lived in the surveyed areas. In this way, a total sample of 2,374 women aged 15-49 years who had a live birth in the three year prior to the interview was selected.

\section{Dependent variables: Utilization of maternal health services}

Two dependent variables (Antenatal care and Place of delivery) are used in this study in order to examine the utilization of maternal health services. According to the WHO recommendation, a woman should visit with health personnel at least four times during her pregnancy to protect from complication. Thus, antenatal care has two categories: inadequate visit (less than four) coded as 0 and adequate visit (four or more) coded as 1 . Place of Delivery is another dependent variable which is defined whether the place of delivery for last birth was delivered at home or health facility. The category, health facility, includes all types of institutional deliveries such as government hospitals, health centers, health posts, private hospitals, clinics, and nursing homes. Births delivered at home were coded as 0 and births delivered at a health facility were coded as 1 .

\section{Independent variables: Women's autonomy}

Women's autonomy is multidimensional and it is very difficult to measure. It comprises the entire complex of interactions, roles, rights and status that surround being male versus being female in a given society or culture (Mason, 1998). However, this study tries to measure it in the domestic sphere.

The NDHS, 2011 asks several questions on these indicators. The variable, women's participation in household decision making, comes from the question "Who in your family makes decisions about (1) healthcare for yourself, (2) large household purchases, (3) visits to family and/or relatives, and (4) uses of contraception.

In Nepal, there is a strong sense of family "togetherness" and individual identity is closely tied to the family, so decisions often involve complex negotiations (Acharya M. and Bennett L, 1981). Measuring whether a woman's joint decision in the final decision making is therefore a more suitable measure than whether or not she is the sole decision maker. Therefore, whether the women had made final decision on household matters, variables are recoded into three categories of 'self-decision (has the final say alone)' coded as 2, 'Joint decision with husband or others (respondent joint decision with husband or other family member)' coded as 1 , and 'no decision (husband only and someone else has the final say)' coded as 0 .

Control over earning refers to whether the women can control over their earning or not. The NDHS, 2011 asked women question relating to control over earnings "Who mainly decides how 
the money that you earn will be used?" Answers were categorized into a dichotomous variable: no control over earnings (including those who worked without earning any cash income), coded as 0 and control over earnings (alone or jointly) coded as 1 . Other nine indirect independent variables are also used to show the effects of women's autonomy on utilization of maternal health care services before and after controlling these variables.

Variables used in the study are

\begin{tabular}{|l|l|l|}
\hline \multicolumn{1}{|c|}{$\begin{array}{c}\text { Indirect independent variables } \\
\text { (Proxy) }\end{array}$} & \multicolumn{1}{|c|}{$\begin{array}{c}\text { Direct independent variable } \\
\text { (Direct autonomy variables) }\end{array}$} & \\
\hline - Age & Household decision making & \\
- Age at marriage & power on & \\
- Birth planning status & - Own health care & - Antenatal care \\
- Number of living children & - Large household purchase \\
- Education of women & - Visit to family or relatives & - Delivery care \\
- Occupation of husband & Decision on contraception use & \\
- Household economic status & Control over own earnings & \\
- Exposure to media & \multicolumn{2}{|}{} \\
\hline
\end{tabular}

\section{METHOD OF DATA ANALYSIS}

Data is analyzed using SPSS software (version 16), and multivariate logistic regression analysis was done to identify the independent effects of explanatory variables on the outcomes of interest. To control the confounding influence of the indirect independent variables, two models were fitted for both dependent variables to identify the independent relationship between the variables of autonomy and utilization of maternal health care services. The first model in both dependent variables includes all autonomy variables taken together without controlling other indirect independent variable's effects. The second model includes women's autonomy indicators along with all other indirect independent variables (such asage, age at marriage, birth planning status, number of living children, education of women, education of husband, occupation of women, household economic status, and exposure to media) that may have influence on utilization of maternal health care services. After assessing correlation among the independent variables, results of correlation matrix shows that no variable were highly correlated. Thus, all variables are used in logistic regression analysis.

\section{RESULTS}

Table 1 shows the odds ratio (OR) for utilization of maternal health care services (adequate ANC visits and delivery at health facilities) with respect to women's autonomy. Women who were involved in decision making regarding their own health had significantly more likely to receive antenatal care than those who were not involved. Women who had some control over their earning were more likely to receive antenatal care compared to those women who had no command over their earning. Women who make joint decision on their own health care were significantly more likely to have adequate ANC visits $(\mathrm{OR}=2.009)$. The association became weaker but still remained 
significant even when adjusted for all other indirect independent variables included in Model II $(\mathrm{OR}=1.398)$. Similarly, women who make self-decision on their own health care were significantly more likely to have adequate ANC visits $(\mathrm{OR}=1.667)$. But the association became insignificant when adjusted for all other indirect independent variables included in Model II. Women who have some control over their earning were more likely to receive antenatal care compared to those women who had no command over their earning (from adjusted model $\mathrm{OR}=1.656$ ). In the similar trend, women who make self or joint decision on the use of contraceptive method are more likely to receive antenatal care.

Women who make joint decision on their own health care are more likely to deliver their baby in medical institution than that of women who have no decision about it $(\mathrm{OR}=1.572)$. Similarly, women who make self-decision on large household purchase are 1.7 times more likely to give birth in medical institution than that of women who have no decision about it. Women who make self or joint decision on using contraception and can control their earning are 1.6 times more likely to deliver their baby in medical institution.

Table 1: Odds ratios from logistic regression to measure the effect of women's autonomy on utilization of maternal health care services $(n=2,374)$

\begin{tabular}{|c|c|c|c|c|}
\hline \multirow[t]{2}{*}{ Autonomy variables } & \multicolumn{2}{|c|}{$\begin{array}{l}\text { Receiving adequate antenatal } \\
\text { care }\end{array}$} & \multicolumn{2}{|c|}{$\begin{array}{l}\text { Giving birth in medical } \\
\text { institutions }\end{array}$} \\
\hline & ModelI & Model II & ModelI & ModelII \\
\hline \multicolumn{5}{|l|}{ Decision on women's own health care } \\
\hline Joint decision with husband or others & $2.009 * * *$ & $1.398 * *$ & $2.040^{* * *}$ & $1.572 * * *$ \\
\hline Self-decision & $1.667 * * *$ & 1.234 & 1.238 & 1.009 \\
\hline $\begin{array}{l}\text { Decision on major household purchase } \\
\text { No decision (R) }\end{array}$ & & & & \\
\hline Joint decision with husband or others & 0.915 & 1.287 & 0.802 & 0.949 \\
\hline Self-decision & 1.161 & 1.565 & 1.405 & $1.789 * * *$ \\
\hline $\begin{array}{l}\text { Decision on visit to relatives } \\
\text { No decision (R) }\end{array}$ & & & & $\checkmark$ \\
\hline Joint decision with husband or others & $0.696 * * *$ & 0.762 & 0.747 & 0.879 \\
\hline Self-decision & 0.838 & 0.919 & 0.823 & 0.913 \\
\hline $\begin{array}{l}\text { Decision on contraceptive use } \\
\text { No decision (R) }\end{array}$ & & & & \\
\hline Joint decision with husband & $1.841^{* *}$ & $1.955 *$ & $1.425^{* *}$ & $1.656^{*}$ \\
\hline Self-decision & 1.416 & 1.704 & 1.357 & $1.681^{*}$ \\
\hline $\begin{array}{l}\text { Control over own earning } \\
\text { No control over earning (R) }\end{array}$ & & & & \\
\hline Control over earning & $1.425 *$ & $1.656^{*}$ & 1.357 & $1.681^{*}$ \\
\hline
\end{tabular}

Note: Model I is the outcomes of autonomy variables only and Model II is the outcomes of controlling other indirect independent variables such asage of women, age at marriage, birth planning status, number of living children, education of women, education of husband, occupation of women, household economic status, and exposure to media.

$*, * * \& * * *$ indicate values are significant at $10 \%, 5 \%$ \& $1 \%$ level respectively. 


\section{DISCUSSION}

This study assessed the effect of women's autonomy on the utilization of maternal health care service among rural women in Nepal. Two measures of maternal health care service utilization were considered: Adequate antenatal care (4 or more ANC visit) and, delivery at health facility. Women's autonomy is measured by women's household decision-making autonomy (own health care, large household purchases, visits to family or relatives and decision on using contraception) and control over own earning. Many studies and policies have been based on the assumption that if women were more involved in household decision making and had more control over financial resources, they would be more likely to use health services and, hence, to have better health outcomes. However, results from this study reveal a more complex picture, showing diverse relationships between the outcomes of interest and the indicators of women's autonomy.

It was theorized that women with some decision making power in the household are better able to communicate with their family members and, therefore, are capable of demanding care for themselves when needed (Bhatia \& Cleland, 1995; Hogan et al., 1999). Among the five areas of women's decision making autonomy, only two decisions making regarding own health care and using contraception have positive effects on the use of antenatal care services and all of the decisions except decision on visit to relatives have significant positive effects on the delivered their baby in medical institution. It is similar to the study carried out in India (Basu, 1993; Bloom, Wypij, \& Gupta, 2001). This suggest that if women were conscious in own health care, involved in large household purchase, and involved in decision on using contraception, they would more likely to use maternal health care. However, in other areas of decision making, this study did not show any influence on the use of maternal health services. And it was also hypothesized that women who had some control over their earning are more likely to utilize maternal health services than that of women with no control over their earning. This relationship was statistically significant with both dependent variables (i.e. antenatal care and delivery care).

\section{CONCLUSIONS}

This study provides important insights about association between women's autonomy and maternal health care utilization in Nepal. Fewer women had made the household decisions in Nepal and women's role in decision making varies with the types of decisions. However, it seems that household decision making power of Nepalese women is quite low and respondent's husband or any other person of the households had higher level of power in each area. Women's autonomy in decision making regarding health care and control over earning is significantly associated with the use of antenatal and delivery care services because financial sufficiency is must at that period. The findings of this study entail that women's autonomy is needed to be improved for the better use of maternal health care services which results in low morbidity and mortality among the women of reproductive age group. Participation of women in household decision making and health care decision will increase the health care utilization during pregnancy and delivery leading to better maternal and child health outcome.

\section{Limitations}

This study has mainly used data from Nepal Demographic and Health Surveys 2011. Therefore, the variables used in the study may deserve criticism for their limited scope of defining women's 
autonomy. Since, this study is limited to secondary data; assessment of the processes of utilization of maternal health care services impact of women's autonomy is beyond the scope of this research. Utilization of maternal health services is influenced by both demand and supply factors. But this study is only limited to the demand side factors and it is not considered the supply side factors like cost, availability, and distance. A qualitative approach of examining the effects of women's autonomy on utilization of maternal health services is not use in this study.

\section{Acknowledgments}

The author thanks MEASURE DHS+ for providing access to the data.

\section{REFERENCES}

Acharya, M.,\& Bennett, L. (1981). The rural women of Nepal: An aggregate analysis and summary of eight village studies. Kathmandu: Centre for Economic Development and Administration.

Basu, A.M. (1993). Culture, the status of women and demographic behaviour illustrated with the case of India. Oxford: Clarendon Press.

Batliwala, S. (1994). The meaning of women's empowerment: New concepts from action.In Gita Sen, Adriennne Germain,\& Lincoln Chen (eds.) Population policies reconsidered: Health, empowerment and rights. Cambridge, Mass: Harvard University Press, 126-138.

Bhatia, J.,\& Cleland J. (1995). Determinants of maternal care in a region of South India. Health Transition Review, 5 (2), 127-142.

Bloom, S.S., Wypij, D.,\&Gupta, M.D. (2001). Dimensions of women's autonomy and the influence on maternal health care utilization in a North Indian city. Demography, 38 (1),67-78.

Dyson, T.S.,\&Moore, M. (1983). On kinship structure, female autonomy and demographic behaviour in India. Population and Development Review,9(1),35-60.

Jejeebhoy, S.J. (2000a). Women's autonomy in rural India: Its dimensions, determinants and the influence of context. In Harriet B. Presser and Gita Sen (eds.) Women's empowerment and demographic processes: Moving beyond Cairo. New York: Oxford University Press, 204-238.

(2000b). Women's autonomy and reproductive behaviour in India. In Zeba Ayesha Sathar,\& James F. Phillips (eds.) Fertility transition in South Asia. Oxford: Oxford University Press, 221-241.

Mason, K.O. (1998). Wives' economic decision-making power in the family: Five Asian countries. In K.O. Mason (ed.) The changing family in comparative perspective: Asia and the United States. Honolulu: East-West Center, 105-133.

Ministry of Health (MoH) (1994). Safe motherhood policy. Kathmandu: MoH.

(1991).New national health policy. Kathmandu: MoH.

Ministry of Health and Population (MoHP). (1996). National maternity care guidelines, Nepal. Kathmandu: MoHP. 
Ministry of Health and Population (MoHP) [Nepal], New ERA,\& ICF International Inc. (2012). Nepal demographic and health survey 2011. Kathmandu, Nepal\& Calverton, Maryland: MoHP, New ERA,\& ICF International.

Ministry of Health and Population (MoHP) [Nepal], New ERA,\& Macro International Inc. (2007). Nepal demographic and health survey 2006. Kathmandu: MoH, New ERA,\& Macro International Inc.

Radcliffe-Brown, A.R. (1952). Structure and function in primitive society. London: Cohen and West. 